\title{
Cross-Cultural Adaptation and Validation of the Persian Version of the 24-Item Early-Onset Scoliosis Questionnaire
}

\author{
Mahmood Esfandiari ${ }^{1}$, Taher Babaee ${ }^{1}$, Mojtaba Kamyab ${ }^{1}$, Mohammad Kamali ${ }^{2}$, \\ Hiroko Matsumoto ${ }^{3,4}$, Hassan Ghandhari ${ }^{5}$, Michael G. Vitale ${ }^{3}$ \\ ${ }^{1}$ Rehabilitation Research Center, Department of Orthotics and Prosthetics, School of Rehabilitation Sciences, Iran University of Medical Sciences, Tehran, Iran \\ ${ }^{2}$ Rehabilitation Research Center, School of Rehabilitation Sciences, Iran University of Medical Sciences, Tehran, Iran \\ ${ }^{3}$ Division of Pediatric Orthopaedic Surgery, Department of Orthopaedic Surgery, Irving Medical Center, Columbia University, New York, NY, USA \\ ${ }^{4}$ Department of Epidemiology, Mailman School of Public Health, Columbia University, New York, NY, USA \\ ${ }^{5}$ Bone and Joint Reconstruction Research Center, Shafa Orthopedic Teaching Hospital, Iran University of Medical Sciences, Tehran, Iran
}

Study Design: Cross-sectional study.

Purpose: To translate and culturally adapt the original English version of the 24-item Early-Onset Scoliosis Questionnaire (EOSQ-24) to the Persian language as well as assess its reliability and validity.

Overview of Literature: Early-onset scoliosis (EOS) patients with progressive curves require active treatments, such as serial elongation-derotation-flexion casting, bracing, and surgery, which are stressful and expensive. In order to evaluate the impact of EOS and its treatment strategies, it is important to consider the patients and their parents' quality of life as the clinical and radiological parameters. The EOSQ-24 is a parent-based measure that evaluates the health-related quality of life of patients with EOS and their caregivers/parents. Similar to other widely used questionnaires, EOSQ-24 needs to be translated into other languages to make it usable in populations from different cultures and societies.

Methods: We evaluated the translation and back translation of the EOSQ-24 and made the required revisions as per the analysis performed by the expert committee and an international guideline to adapt it for use in this study. Thereafter, we recruited 100 EOS patients in order to evaluate its reliability and validity. The reliability was assessed with internal consistency. Convergent validity was assessed by comparing the scores of the EOSQ-24 and the 22-item Scoliosis Research Society Questionnaire (SRS-22r). Finally, the known groups validity was assessed as per patient's sex, curve magnitude, and treatment type.

Results: The Persian EOSQ-24 demonstrated very good internal consistency (Cronbach's $\alpha=0.88$ ). All the items had an acceptable corrected item-total correlation (>0.3). Further, the EOSQ-24 and the SRS-22r scores $(p<0.001)$ were significantly correlated. The EOSQ-24 could discriminate patients with different curve magnitudes.

Conclusions: The Persian EOSQ-24 can serve as a disease-specific instrument with strong validity and reliability in the evaluation of EOS patients. Its applicability in other Persian-speaking countries and regions of the world needs to be investigated further.

Keywords: Questionnaire; Scoliosis; Reliability; Validity; Translation

Received Sep 15, 2020; Revised Oct 26, 2020; Accepted Nov 9, 2020

Corresponding author: Taher Babaee

Rehabilitation Research Center, Department of Orthotics and Prosthetics, School of Rehabilitation Sciences, Iran University of Medical Sciences, Madadkaran Avenue, Shahnazari St., Madar Square, Mirdamad Blvd., Tehran, Iran

Tel: +98-21-2222-0947 (Ext. 267), Fax: +98-21-2222-0946, E-mail: babaee.t@iums.ac.ir 


\section{Introduction}

Early-onset scoliosis (EOS) is defined as an abnormal spine curve of $>10^{\circ}$ with an onset age of $<10$ years [1]. Congenital anomalies of the vertebrae, neuromuscular, and syndromic conditions, and unknown causes are common causal reasons. If left untreated, EOS may cause severe spinal deformities and cardiorespiratory problems, increasing the mortality risk [2-4]. Pediatric patients with EOS and their caregivers experience several challenges owing to progressive spine deformity and the possibility of lung dysfunction [5]. Thus, a comprehensive view of the condition of EOS patients and their families is important.

The most common treatments for pediatric EOS patients are serial casting [6], brace wearing [7], and surgery [8]. Complications that can be treated non-surgically are skin lesions, chest deformities, cardiorespiratory problems, and kidney dysfunction [9]. Complications related to surgical procedures include infection, instrumentation failure, long-term hospital stay, and neurodevelopmental issues caused by repeated anesthesia administration [1012].

Generally, EOS patients with progressive curves need long-term treatment that is stressful and expensive; further, it may affect different life aspects of the patients and their parents. Therefore, as clinical and radiological parameters, the health-related quality of life (HRQoL) variables of EOS patients should also be considered to evaluate the outcomes of the disease and its treatment types.

The 24-item Early-Onset Scoliosis Questionnaire (EOSQ-24) is a parent-based instrument designed by Matsumoto et al. [13] for assessing the HRQoL of EOS patients and their caregivers. Previous research has demonstrated that EOSQ-24 is a valid outcome measure for evaluating the quality of life (QoL) of EOS patients [1420].

Similar to other widely used questionnaires, the EOSQ24 requires to be translated into other languages for use in populations from different cultures and societies. This increases the possibility of international communication and allows researchers to compare the results of their studies. Currently, the EOSQ-24 is available in Chinese [21], Arabic [17], Norwegian [18], Dutch [15], Turkish [20], Spanish [19], German [16], and Brazilian Portuguese [14] versions.

Thus, this study aimed to translate the EOSQ-24 into Persian and investigate its validity and reliability.

\section{Materials and Methods}

The ethics committee of Iran University of Medical Sciences approved the study protocol (no., IR.IUMS. REC.1398.791). Before initiating the study, we contacted the EOSQ-24 developers via email and obtained their approval for translation.

The study was performed in the following two phases: (1) the translation and cross-cultural adaptation of the EOSQ-24 into the Persian version (P-EOSQ-24) (development phase), and (2) the validity and reliability analysis of the P-EOSQ-24 (validation phase).

\section{Translation and cross-cultural adaptation phase}

The translation and cultural adaptation process were based on the guidelines described by Beaton et al. [22] and performed in five stages. In the first stage, two professional Persian language translators fluent in the English language translated the original English version of the EOSQ-24 into Persian language. In the second stage, the two translations were merged into a single Persian version with a consensus among the two translators and the research team. In the third stage, two independent professional bilingual translators who were fluent in English and Persian translated this Persian version into English (the back translation stage). After resolving the discrepancies between the two translations, we sent the back translation to the original EOSQ-24 developers. After obtaining their approval, an expert committee that comprised all the translators, three certified orthotists, a methodologist, and a spine surgeon reviewed all the translations to prepare a pre-final Persian version of the EOSQ-24. The goal of this stage was to achieve a cross-cultural equivalence, including semantic, idiomatic, and experiential equivalence.

Thereafter, the pre-final Persian version of the EOSQ24 was used for 30 Persian-speaking parents of children with EOS at the Department of Orthopedic Surgery, the Division of Spine Surgery, Shafa Yahyaiian Hospital, Tehran, Iran. The parents were asked to rate each item on a 5-point Likert scale with respect to the clarity and intelligibility of each item. After reviewing and calculating the scores, we found that most parents had understood the questions correctly. The percentage of concession on all items was $>80 \%$. 


\section{Reliability and validity-testing phase}

To evaluate the reliability and validity of the P-EOSQ-24, we administered it to 100 Persian-speaking parents of pediatric patients with EOS. In addition, we asked these parents to fill the parent form of the Persian 22-item Scoliosis Research Society questionnaire (SRS-22r) to test the convergent validity of the EOSQ-24. Data were collected from two spine centers in Tehran, Iran in the period from September 2019 to March 2020. The parents of all pediatric subjects signed a written consent form before the subjects were enrolled in the study.

\section{Questionnaires}

\section{1) The EOSQ-24}

The EOSQ-24 is a parent-based questionnaire that comprises 24 items in 4 domains and 11 sub-domains [13]. The HRQoL domain measures the HRQoL of pediatric patients with EOS and collects information about general health (two items), pain/discomfort (two items), pulmonary function (two items), transfer (one item), physical function (three items), daily living (two items), fatigue/ energy level (two items), and emotion (two items). Parental burden (five items) and financial burden (one item) are separate domains that measure the burden on parents, and the satisfaction (two items) domain measures the satisfaction level of the pediatric patients and their parents. Each question is answered using a 5-point Likert scale that ranges from 1 to 5 , with 1 indicating the worst condition and 5 indicating the best condition. Scores for each domain and sub-domain range from 0 to 100 . Normative data based on age were also collected.

\section{2) The SRS-22r}

The SRS-22r questionnaire is a self-reported instrument that is used to assess the QoL of patients with idiopathic scoliosis [23]. This questionnaire includes 22 items in five subscales, including function/activity (five items), pain (five items), self-image (five items), mental-health (five items), and satisfaction with the management (two items). For each item, the parents are required to mark their perception regarding their child's condition on a 5-point Likert scale that ranged from 1 to 5, with 1 indicating the worst condition and 5 indicating the best condition. Therefore, the score of each subscale ranged from 5 to 25 (except for the satisfaction subscale that ranged from 2 to
10). The total score of the SRS-22r ranged from 22 to 110.

\section{Reliability analyses}

We evaluated the reliability by calculating the internal consistency of the EOSQ-24. "Corrected item-total correlation" was calculated in order to evaluate the correlation between each item and the entire questionnaire. We calculated "Cronbach's $\alpha$ if item deleted" to determine whether the Cronbach's $\alpha$ value improved after deleting a question.

\section{Validity analyses}

1) Convergent validity

Convergent validity refers to the relationship between the scores of two different instruments that examine a single variable [24]. In this study, we asked all participants to complete the P-EOSQ-24 and the parent form of the SRS$22 \mathrm{r}$ in order to evaluate convergent validity [25].

\section{2) Known groups validity}

Known groups validity is a type of construct validity that refers to the ability of an instrument to distinguish individuals who are known to have different characteristics [26]. In order to evaluate the known groups validity, we supposed that the total scores of the P-EOSQ-24 are different as per the patient's sex, curve magnitude, and treatment type.

\section{Floor and ceiling effects}

The floor and ceiling effects indicate the limitation of an instrument to assess the condition of the patients being studied; this is related to the content validity. Values $<30 \%$ of the floor and ceiling effects are considered acceptable [19]. In our study, the floor and ceiling effects were calculated according to the frequency of the minimum and maximum scores for a question ( 1 or 5 ).

\section{Statistical analyses}

We used the IBM SPSS ver. 20.0 (IBM Corp., Armonk, NY, USA) for our analyses. The graphs were created using the GraphPad Prism software for Windows 8 (GraphPad Prism software Inc., San Diego, CA, USA). We reported the descriptive data as mean values, standard deviation 
values, missing answers, interquartile ranges, as well as the ceiling and floor effects. Cronbach's a coefficient was calculated in order to evaluate the internal consistency of the EOSQ-24. A value of $0.70 \leq a \leq 0.95$ for Cronbach's $\alpha$ indicates a satisfactory internal consistency [27]. We used the corrected item-total correlation in order to determine the relationship between each item of the EOSO-24 and its total score. A value of $>0.3$ showed that the item had an acceptable interrelationship with the questionnaire [27].

Spearman's correlation coefficient was used for evaluating the relationship between the EOSQ-24 and SRS-22r for convergent validity. Moreover, a sub-group analysis using an independent sample $t$-test was used to evaluate the known groups validity of the EOSQ-24 with respect to the patient's sex, curve magnitude, and treatment type. The significance level was indicated by a $p$-value $<0.05$, with $95 \%$ confidence intervals (CIs) for all correlation coefficients.

\section{Results}

\section{Study participants}

At the pre-final testing of the P-EOSQ-24, all pediatric patients (19 females and 11 males) had idiopathic scoliosis, and their average age and curve magnitude were $6.30 \pm 2.16$ years and $35.46^{\circ} \pm 8.56^{\circ}$, respectively. Of these,

\begin{tabular}{|c|c|}
\hline Characteristic & Value \\
\hline \multicolumn{2}{|l|}{ Gender $(\%)$} \\
\hline Male & $39(39.0)$ \\
\hline Female & $61(61.0)$ \\
\hline \multicolumn{2}{|l|}{ Age (mo) } \\
\hline At diagnosis & $72(24-47)$ \\
\hline At initiation of treatment & $72(36-105)$ \\
\hline Cobb angle $\left({ }^{\circ}\right)$ & $40(33-48)$ \\
\hline \multicolumn{2}{|l|}{ Intervention (\%) } \\
\hline Brace treatment & $81(81.0)$ \\
\hline Surgical treatment & $19(19.0)$ \\
\hline \multicolumn{2}{|l|}{ Diagnosis } \\
\hline Idiopathic & $94(94.0)$ \\
\hline Congenital & $2(2.0)$ \\
\hline Syndromic & $4(4.0)$ \\
\hline
\end{tabular}

Values are presented as number (\%) or median (interquartile range). seven patients had received surgical intervention, and 23 had undergone brace treatment. In the reliability and validity-testing phase, among the 100 EOS patients, 81

Table 2. Internal consistency analysis of the EOSQ-24

$\begin{array}{lccc}\text { EOSQ-24 domains Mean } \pm \text { SD } & \begin{array}{c}\text { Corrected } \\ \text { item-total } \\ \text { correlation }\end{array} & \begin{array}{c}\text { Cronbach's } \\ \alpha \text { if item } \\ \text { deleted }\end{array} & \begin{array}{c}\text { Cronbach's } \\ \text { domain }\end{array} \\ \text { General health } & & & 0.69\end{array}$

\begin{tabular}{ccccc}
01 & $3.69 \pm 1.09$ & 0.76 & 0.91 & \\
02 & $4.49 \pm 0.83$ & 0.69 & 0.93 & \\
\hline Pain & & & & 0.85 \\
03 & $4.01 \pm 0.97$ & 0.85 & 0.96 & \\
04 & $4.12 \pm 0.92$ & 0.84 & 0.96 & \\
\hline
\end{tabular}

Pulmonary function $\quad 0.63$

\begin{tabular}{|c|c|c|c|c|}
\hline 05 & $4.18 \pm 0.96$ & 0.72 & 0.89 & \\
\hline 06 & $4.52 \pm 0.73$ & 0.64 & 0.92 & \\
\hline Transfer & & & & NA \\
\hline 07 & $4.30 \pm 1.04$ & 0.67 & 0.80 & \\
\hline Physical function & & & & 0.85 \\
\hline 08 & $3.68 \pm 1.09$ & 0.77 & 0.92 & \\
\hline 09 & $3.51 \pm 1.05$ & 0.75 & 0.92 & \\
\hline 010 & $3.53 \pm 1.04$ & 0.81 & 0.91 & \\
\hline Daily living & & & & 0.49 \\
\hline 011 & $3.48 \pm 1.05$ & 0.53 & 0.90 & \\
\hline 012 & $3.58 \pm 1.41$ & 0.64 & 0.86 & \\
\hline Fatigue & & & & 0.75 \\
\hline 013 & $3.94 \pm 0.99$ & 0.76 & 0.94 & \\
\hline 014 & $3.71 \pm 1.01$ & 0.76 & 0.94 & \\
\hline Emotion & & & & 0.87 \\
\hline 015 & $3.91 \pm 1.09$ & 0.88 & 0.96 & \\
\hline 016 & $4.22 \pm 0.95$ & 0.86 & 0.97 & \\
\hline Parental impact & & & & 0.84 \\
\hline 017 & $3.25 \pm 1.16$ & 0.56 & 0.90 & \\
\hline 018 & $3.98 \pm 1.16$ & 0.79 & 0.86 & \\
\hline 019 & $3.55 \pm 1.17$ & 0.78 & 0.87 & \\
\hline 020 & $4.30 \pm 1.02$ & 0.69 & 0.88 & \\
\hline 021 & $4.17 \pm 0.84$ & 0.59 & 0.89 & \\
\hline Financial impact & & & & NA \\
\hline 022 & $2.92 \pm 1.26$ & 0.63 & 0.77 & \\
\hline Satisfaction & & & & 0.67 \\
\hline 023 & $3.41 \pm 0.81$ & 0.69 & 0.92 & \\
\hline 024 & $3.55 \pm 0.83$ & 0.70 & 0.92 & \\
\hline
\end{tabular}

EOSQ-24, 24-item Early-Onset Scoliosis Questionnaire; SD, standard deviation; 0 , question; NA, not applicable. 
had undergone brace treatment, and 19 had received surgical treatment (Table 1). All the patients were recruited during their routine office visits. The P-EOSQ-24 has been included in Supplement 1.

\section{Reliability}

The P-EOSQ-24 possesses very good internal consistency (Cronbach's $\alpha$ of 0.886 ; range, 0.56-0.96). The Cronbach's a per sub-domain ranged from 0.49 in the "daily living" sub-domain to 0.87 in the "emotion" sub-domain (Table

Table 3. Results of Pearson correlation coefficients between subscales of the EOSQ-24 and the SRS-22

\begin{tabular}{llll} 
EOSO-24 & \multicolumn{1}{c}{ SRS-22 } & \multicolumn{1}{c}{$r$} & $95 \% \mathrm{Cl}$ \\
\hline Pain & Pain & $0.67^{* * *}$ & $0.48-0.78$ \\
Transfer & Function & $0.44^{* * *}$ & $0.23-0.62$ \\
\hline Physical function & Function & $0.54^{* * *}$ & $0.33-0.70$ \\
Daily living & Function & $0.53^{* * *}$ & $0.35-0.68$ \\
\hline Emotion & Mental health & $0.58^{* * *}$ & $0.44-0.70$ \\
\hline
\end{tabular}

EOSO-24, 24-item Early-Onset Scoliosis Questionnaire; SRS-22, 22-item Scoliosis Research Society Questionnaire; $r$, correlation coefficient; $\mathrm{Cl}$, confidence interval.

${ }^{* * *} p<0.001$ is statistically significant.

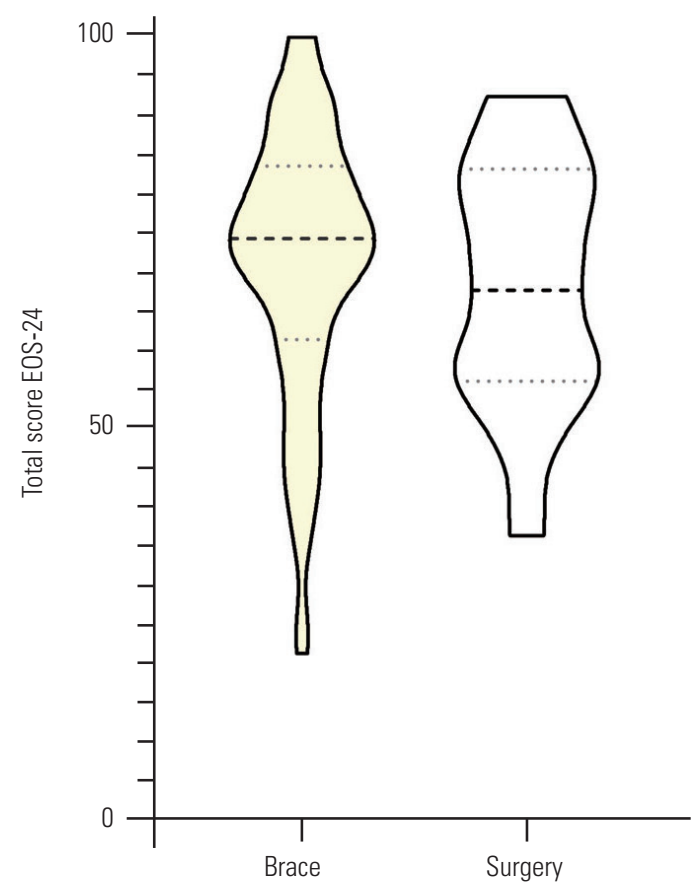

Fig. 1. Violin plots of total 24-item Early-Onset Scoliosis Questionnaire (EOSO24) scores between patients with different types of treatment. The middle, lower, and upper horizontal dash lines demonstrate the median, 1st quartile, and 3rd quartile values, respectively $(p>0.05)$.

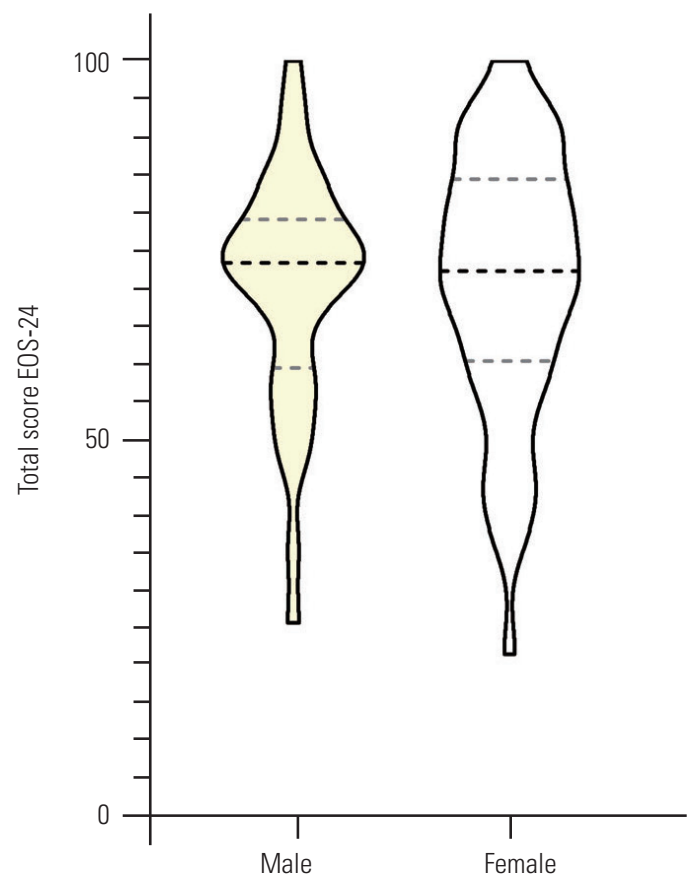

Fig. 2. Violin plots of total 24-item Early-Onset Scoliosis Questionnaire (EOSQ24) scores between males and females. The middle, lower, and upper horizontal dash lines demonstrate the median, 1st quartile, and 3rd quartile values, respectively $(p>0.05)$.

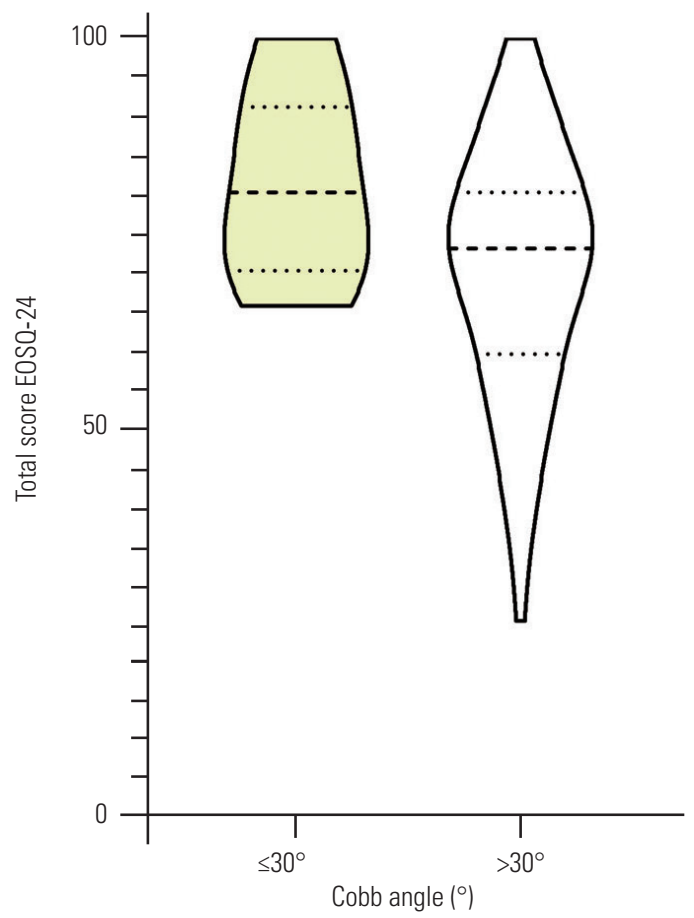

Fig. 3. Violin plots of total 24-item Early-Onset Scoliosis Questionnaire (EOSQ24) scores between patients with curve magnitude of $\leq 30^{\circ}$ and $>30^{\circ}$. The middle, lower, and upper horizontal dash lines demonstrate the median, $1 \mathrm{st}$ quartile, and 3rd quartile values, respectively $(p<0.05)$. 
Table 4. Descriptive statistics of each item and domain of the EOSQ-24

\begin{tabular}{|c|c|c|c|c|c|}
\hline Variable & Median (IQR) & Mean \pm SD & Floor $(\%)$ & Ceiling (\%) & Missing (\%) \\
\hline \multicolumn{6}{|c|}{ General health } \\
\hline 01 & $4.00(3.00-5.00)$ & $3.73 \pm 1.06$ & 4 (3.2) & $25(20.0)$ & 1 \\
\hline 02 & $5.00(4.00-5.00)$ & $4.53 \pm 0.77$ & $1(0.8)$ & $65(52.0)$ & 2 \\
\hline \multicolumn{6}{|l|}{ Pain } \\
\hline 03 & $4.00(3.00-5.00)$ & $4.05 \pm 0.97$ & $9(7.2)$ & $39(31.2)$ & 0 \\
\hline 04 & $4.00(4.00-5.00)$ & $4.18 \pm 0.91$ & $1(0.8)$ & 42 (33.6) & 0 \\
\hline \multicolumn{6}{|c|}{ Pulmonary function } \\
\hline 05 & $4.00(3.00-5.00)$ & $4.22 \pm 0.87$ & $1(0.8)$ & $50(40.0)$ & 1 \\
\hline 06 & $5.00(4.00-5.00))$ & $4.56 \pm 0.71$ & $2(1.6)$ & $64(51.2)$ & 2 \\
\hline \multicolumn{6}{|l|}{ Transfer } \\
\hline 07 & $5.00(4.00-5.00)$ & $4.35 \pm 1.00$ & $3(2.4)$ & $58(46.4)$ & 3 \\
\hline \multicolumn{6}{|c|}{ Physical function } \\
\hline 08 & $4.00(3.00-5.00)$ & $3.69 \pm 1.04$ & $3(2.4)$ & $28(22.4)$ & 1 \\
\hline 09 & $3.00(3.00-4.00)$ & $3.50 \pm 1.02$ & $5(4.0)$ & $20(16.0)$ & 2 \\
\hline 010 & $3.00(3.00-4.00)$ & $3.52 \pm 0.99$ & $3(2.4)$ & $23(18.4)$ & 2 \\
\hline \multicolumn{6}{|c|}{ Daily living } \\
\hline 011 & $3.00(3.00-4.00)$ & $3.55 \pm 091$ & $5(4.0)$ & $20(16.0)$ & 1 \\
\hline 012 & $4.00(3.00-5.00)$ & $3.80 \pm 1.28$ & $13(10.4)$ & $40(32.0)$ & 0 \\
\hline \multicolumn{6}{|l|}{ Fatigue } \\
\hline 013 & $4.00(3.00-5.00)$ & $4.05 \pm 0.86$ & $1(0.8)$ & $35(28.0)$ & 0 \\
\hline 014 & $4.00(3.00-5.00)$ & $3.77 \pm 0.90$ & $3(2.4)$ & $25(20.0)$ & 2 \\
\hline \multicolumn{6}{|l|}{ Emotion } \\
\hline 015 & $4.00(3.00-5.00)$ & $3.97 \pm 1.05$ & $1(0.8)$ & $40(32.0)$ & 0 \\
\hline 016 & $5.00(3.00-5.00)$ & $4.26 \pm 0.95$ & $4(3.2)$ & 54 (43.2) & 2 \\
\hline \multicolumn{6}{|c|}{ Parental burden } \\
\hline 017 & $3.00(2.00-4.00)$ & $3.32 \pm 1.13$ & $5(4.0)$ & $18(14.4)$ & 0 \\
\hline 018 & $4.00(3.00-5.00)$ & $4.07 \pm 1.12$ & $4(3.2)$ & $43(34.4)$ & 0 \\
\hline 019 & $4.00(3.00-4.00)$ & $3.57 \pm 1.16$ & $8(6.4)$ & $20(16.0)$ & 0 \\
\hline 020 & $5.00(4.00-5.00)$ & $4.98 \pm 5.78$ & $2(1.6)$ & $60(48.0)$ & 0 \\
\hline 021 & $4.00(4.00-5.00)$ & $4.21 \pm 0.82$ & $6(4.8)$ & 39 (31.2) & 0 \\
\hline \multicolumn{6}{|c|}{ Financial burden } \\
\hline 022 & $3.00(2.00-4.00)$ & $2.96 \pm 1.26$ & $15(12.0)$ & $15(12.0)$ & 0 \\
\hline \multicolumn{6}{|c|}{ Satisfaction } \\
\hline 023 & $3.00(3.00-4.00)$ & $3.34 \pm 0.80$ & $10(8.0)$ & $10(8.0)$ & 2 \\
\hline 024 & $4.00(3.00-4.00)$ & $3.57 \pm 0.69$ & $3(2.4)$ & $10(8.0)$ & 0 \\
\hline
\end{tabular}

EOS-24, 24-item Early-Onset Scoliosis Questionnaire; IQR, interquartile range; SD, standard deviation; Q, question.

2). The "corrected item-total correlation" analysis showed that the values of all items were $>0.3$.

\section{Validity}

Table 3 shows the relationships between the total and subdomains of the EOSQ-24 scores and their related domains in the SRS-22r. A significant correlation was observed 
between pain, transfer, physical function, daily living, and emotion sub-domains of the EOSQ-24 with their corresponding domains in the SRS-22r, including pain, function, and mental health $(r, 0.44-0.67 ; p<0.001)$. For known groups validity, there was no significant difference between the patient's sex and treatment type with respect to the mean EOSQ-24 scores (Figs. 1, 2). However, a significant difference was observed between patients with a curve magnitude of $\leq 30^{\circ}$ and those with curve magnitudes of $>30^{\circ}(p<0.05)$ (Fig. 3).

\section{Floor and ceiling effects}

There were significant ceiling effects in the Q2 (65\%) from "general health," Q6 (64\%) from "pulmonary function," and Q20 (60\%) from "parental impact" sub-domains of the questionnaire (Table 4). However, no significant floor effect was observed in our study population.

\section{Discussion}

This study investigated the psychometric properties of the P-EOSQ-24 in EOS patients. As per our findings, the PEOSQ-24 has very good internal consistency and satisfactory convergent and known groups validity.

The results of the Cronbach's a coefficient revealed that the P-EOSQ-24 has very good internal consistency (Cronbach's $\alpha=0.88$ ). Therefore, the P-EOSQ-24 has a homogeneous internal structure for measuring the HRQoL of EOS patients. In the other versions of the EOSQ-24, the Cronbach's a value was $0.88-0.95$ [14-21], consistent with that in the P-EOSQ-24. With respect to the sub-domains of the EOSQ-24, the Cronbach's a value was $>0.7$ in the pain, physical functioning, fatigue, parental status, and emotion sub-domains. However, the value of the Cronbach's $\alpha$ was $<0.7$ for general health, pulmonary function, daily living, and satisfaction sub-domains.

In the Chinese [21], Brazilian Portuguese [14], and Dutch [15] versions, Cronbach's $\alpha$ was $<0.7$ in general health and pulmonary function sub-domains, consistent with our results. In the daily living sub-domain, our results were consistent with those in the Brazilian Portuguese version [14]. However, in the satisfaction domain, our results were different from the results of the other versions of the EOSQ-24. This might be because of the different perspectives of the parents with respect to certain questions in this domain. In our study, most parents (87\%) stated that their child did not have a particular problem in dressing and did it without any challenges (Q11). In Q12, 51\% of the parents reported that their child required the same amount of time to eat the same amount of food as a healthy child. In Q23 from the satisfaction domain, the parents are required to answer a question about their child's satisfaction with his/her ability to perform tasks. However, Q24 seeks to know the parents' satisfaction with their child's ability to perform tasks. In Q23, parents answered this question based on their point of view on behalf of their child. In fact, the patients' perceptions about their child's functional limitations and his/her ability to perform tasks can be different from the child's perspective.

In order to evaluate the convergent validity of the P-EOSQ-24, we compared the corresponding sub-domains of this questionnaire with the P-SRS-22r. The results showed a significant correlation between these two questionnaires ( $r$ range, $0.23-0.78$ ). In the report on the Dutch version [15], the researchers first used the parent form of the SRS22 to evaluate the convergent validity of the EOSQ-24, wherein a strong correlation was obtained between the two questionnaires ( $r$ range, $0.61-0.76$ ). The original version of the SRS-22 was developed for adolescents who had idiopathic scoliosis [23]. However, recently, Li et al. [28] conducted a study on patients with congenital scoliosis and found that except for the satisfaction domain, there was a strong correlation between all the sub-domains of the SRS-22 and EOSQ-24. Therefore, SRS-22 is a good instrument for investigating the convergent validity of the EOSQ-24.

The total score of the P-EOSQ-24 showed that it could discriminate patients with different curve magnitudes. As expected, we found that patients with a lower curve magnitude had superior QoL than those with a higher curve magnitude. This result is in keeping with the results obtained from the Spanish [19], Dutch [15], Norwegian [18], and Arabic [17] versions. However, the P-EOSQ-24 could not discriminate between the patient's sex and treatment type. This may be attributable to the heterogeneity of the patients receiving different surgical and nonsurgical treatments. Similar results were also obtained in the Dutch version [15].

The P-EOSQ-24 had a low percentage of missing response $(<1 \%)$, indicating that it offered sufficient clarity and can be well understood by the parents. There were no floor effects in our population in keeping with the results 
obtained in the Dutch [15], Spanish [19], Norwegian [18], Turkish [20], and Chinese [21] versions. However, in the Arabic version [17], Q17 had a significant floor effect (36.2\%). In the Brazilian Portuguese version [14], Q10 and Q11 showed a significant floor effect (43.4\% and $32.9 \%$, respectively). Similar to previous studies, the ceiling effect was observed in some items of the P-EOSQ-24 [14-17,19-21]. The highest ceiling effect was related to Q2 (general health). The reason might be that the child's health was very important to the parents or because most parents do not consider their children as being unwell. Further, Q5 and Q6 (pulmonary function) and Q7 (transfer) had high ceiling effects, in agreement with the results obtained from the Dutch [15], Spanish [19], Norwegian [18], Brazilian Portuguese [14], and Arabic [17] versions.

There were some limitations to this study. First, most study subjects had idiopathic scoliosis and were ambulatory. Therefore, it was not possible to compare their EOSQ-24 scores with those of non-ambulatory patients and patients with syndromic, congenital, and neuromuscular scoliosis. Second, we did not assess the changes in the EOSQ-24 scores over time and during the treatment periods. Third, we performed this study at only two referral centers for EOS; therefore, its generalizability to the entire Persian-speaking population is unclear. However, about $50 \%$ of the patients came from different regions of Iran for treatment.

Finally, Persian is one of the oldest languages of the world that is still spoken. At the present time, this language is spoken in many regions of the world, including but not limited to several modern Middle-Eastern and Central Asian countries. These countries or many of their regions were once parts of Iran (Persia), a much larger country, including Afghanistan, Pakistan, Uzbekistan, Turkmenistan, Tajikistan, Armenia, Azerbaijan, etc. In all these countries, Persian (Farsi) remains the mother tongue or second language of people residing in certain areas. Currently, there are three standard forms of the Persian language, Tehrani or Farsi (used formally in Iran), Dari Persian (used formally in Afghanistan), and Tajiki Persian (used formally in Tajikistan and myriad people in Uzbekistan) [29]. We performed this study in Iran; all the included participants spoke the Tehrani dialect. Although Persian speakers in Iran, Afghanistan, and Tajikistan can communicate with each other, there might be instances of different understandings of a word or phrase in some dialects or vernaculars owing to geographical and cultural differences (the same problem that is apparent in all English or French language questionnaires that are only provided in the standard dialects, including the original English version of the EOSQ-24). Therefore, the applicability of the EOSQ-24 in the other forms of the Persian language and regions of the world requires further investigation.

\section{Conclusions}

The P-EOSQ-24 can serve as a disease-specific instrument that possesses strong validity and reliability for the evaluation of patients with EOS.

\section{Conflict of Interest}

This study was supported by the research committee of Iran University of Medical Sciences. There is no conflict of interest about the study. No potential conflict of interest relevant to this article was reported.

\section{Acknowledgments}

The authors thank Muhammed Hussein Mousavinasab for editing this text.

\section{Author Contributions}

Conception and design: all authors; data acquisition: Mahmood Esfandiari; analysis of data and drafting of the manuscript: Mahmood Esfandiari, Taher Babaee; supervision: Taher Babaee; and critical revision and final approval of the version to be published: all authors.

\section{Supplementary Materials}

Supplementary materials can be available from https:// doi.org/10.31616/asj.2020.0483. Supplement 1. The PEOSQ-24.

\section{References}

1. Skaggs DL, Guillaume T, El-Hawary R, Emans J, Mendelow M, Smith J. Early onset scoliosis consensus statement, SRS Growing Spine Committee, 2015. Spine Deform 2015;3:107.

2. Fernandes P, Weinstein SL. Natural history of early onset scoliosis. J Bone Joint Surg Am 2007;89 Suppl 
1:21-33.

3. Goldberg CJ, Gillic I, Connaughton O, et al. Respiratory function and cosmesis at maturity in infantileonset scoliosis. Spine (Phila Pa 1976) 2003;28:2397406.

4. Pehrsson K, Larsson S, Oden A, Nachemson A. Long-term follow-up of patients with untreated scoliosis: a study of mortality, causes of death, and symptoms. Spine (Phila Pa 1976) 1992;17:1091-6.

5. Yang S, Andras LM, Redding GJ, Skaggs DL. Earlyonset scoliosis: a review of history, current treatment, and future directions. Pediatrics 2016;137:e20150709.

6. Welborn MC, D’Astous J, Bratton S, Heflin J. Infantile idiopathic scoliosis: factors affecting EDF casting success. Spine Deform 2018;6:614-20.

7. Thometz J, Liu XC. Serial CAD/CAM bracing: an alternative to serial casting for early onset scoliosis. J Pediatr Orthop 2019;39:e185-9.

8. Wu AM, Cheung JPY, Cheung KMC, et al. Minimum 2-year experience with magnetically controlled growing rods for the treatment of early-onset scoliosis: a systematic review. Asian Spine J 2019;13:682-93.

9. Thorsness RJ, Faust JR, Behrend CJ, Sanders JO. Nonsurgical management of early-onset scoliosis. J Am Acad Orthop Surg 2015;23:519-28.

10. Bess S, Akbarnia BA, Thompson GH, et al. Complications of growing-rod treatment for early-onset scoliosis: analysis of one hundred and forty patients. J Bone Joint Surg Am 2010;92:2533-43.

11. Akbarnia BA, Emans JB. Complications of growthsparing surgery in early onset scoliosis. Spine (Phila Pa 1976) 2010;35:2193-204.

12. Beaven A, Gardner AC, Marks DS, Mehta JS, Newton-Ede M, Spilsbury JB. Magnetically controlled growing rods: the experience of mechanical failure from a single center consecutive series of 28 children with a minimum follow-up of 2 years. Asian Spine J 2018;12:794-802.

13. Matsumoto H, Williams B, Park HY, et al. The final 24-item Early Onset Scoliosis Questionnaires (EOSQ-24): validity, reliability and responsiveness. J Pediatr Orthop 2018;38:144-51.

14. De Mendonca RG, Bergamascki LM, Silva KC, et al. Validation of the Brazilian Portuguese version of the 24-item Early-Onset Scoliosis Questionnaire. Global Spine J 2020 Jun 19 [Epub]. https://doi. org/10.1177/2192568220933234.
15. Wijdicks SPJ, Dompeling SD, de Reuver S, Kempen DH, Castelein RM, Kruyt MC. Reliability and validity of the adapted Dutch version of the Early-Onset Scoliosis-24-item Questionnaire (EOSQ-24). Spine (Phila Pa 1976) 2019;44:E965-73.

16. Mladenov K, Braunschweig L, Behrend J, Lorenz HM, von Deimling U, Hell AK. Validation of the German version of the 24-item Early-Onset Scoliosis Questionnaire. J Neurosurg Pediatr 2019 Mar 8 [Epub]. https://doi.org/10.3171/2019.1.PEDS18704.

17. Hanbali Y, Perry T, Hanif A, et al. Reliability and validity of the Arabic version of the Early Onset Scoliosis 24 items Questionnaire (EOSQ-24). SICOT J 2019;5:7.

18. Molland RS, Diep LM, Brox JI, Stuge B, Holm I, Kibsgard TJ. Reliability and construct validity of the adapted Norwegian version of the Early-Onset Scoliosis 24-item Questionnaire. J Am Acad Orthop Surg Glob Res Rev 2018;2:e066.

19. Del Mar Pozo-Balado M, Matsumoto H, Vitale MG, Praena-Fernandez JM, Farrington DM. Reliability and validity of the adapted Spanish version of the Early-Onset Scoliosis-24 Questionnaire. Spine (Phila Pa 1976) 2016;41:E625-31.

20. Demirkiran HG, Kinikli GI, Olgun ZD, et al. Reliability and validity of the adapted Turkish version of the Early-onset Scoliosis-24-item Questionnaire (EOSQ-24). J Pediatr Orthop 2015;35:804-9.

21. Cheung JP, Cheung PW, Wong CK, et al. Psychometric validation of the traditional Chinese version of the Early Onset Scoliosis-24 item Questionnaire (EOSQ-24). Spine (Phila Pa 1976) 2016;41:E1460-9.

22. Beaton DE, Bombardier C, Guillemin F, Ferraz MB. Guidelines for the process of cross-cultural adaptation of self-report measures. Spine (Phila Pa 1976) 2000;25:3186-91.

23. Asher M, Min Lai S, Burton D, Manna B. The reliability and concurrent validity of the Scoliosis Research Society-22 patient questionnaire for idiopathic scoliosis. Spine (Phila Pa 1976) 2003;28:63-9.

24. Carlson KD, Herdman AO. Understanding the impact of convergent validity on research results. Organ Res Methods 2012;15:17-32.

25. Mousavi SJ, Mobini B, Mehdian H, et al. Reliability and validity of the Persian version of the Scoliosis Research Society-22r Questionnaire. Spine (Phila Pa 1976) 2010;35:784-9. 
26. Portney LG, Watkins MP. Concepts of measurement. In: Portney LG, Watkins MP, editors. Foundations of clinical research: application to practice. 3rd ed. Philadelphia (PA): F.A Davis Company; 2015. p. 77118.

27. Terwee CB, Bot SD, de Boer MR, et al. Quality criteria were proposed for measurement properties of health status questionnaires. J Clin Epidemiol 2007;60:34-42.
28. Li Y, Burke MC, Gagnier J, Caird MS, Farley FA. Comparison of EOSQ-24 and SRS-22 scores in congenital scoliosis: a preliminary study. J Pediatr Orthop 2020;40:e182-5.

29. Wikipedia. Persian language [Internet]. San Francisco (CA): Wikimedia Foundation Inc.; c2021 [cited 2020 Oct 26]. Available from: https://en.wikipedia. org/wiki/Persian_language. 